\title{
Dark Energy Survey Hits a Triple
}

\author{
A large galaxy survey releases its three-year observations, providing key \\ cosmological-parameter measurements that have double the precision of \\ those previously released.
}

\section{By Michael Schirber}

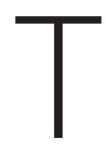

here's more than one way to size up the Universe. The Dark Energy Survey (DES) Collaboration demonstrates this idea by using three statistical measurements of galaxies to pin down values for the dark matter density, the cosmic expansion rate, and other parameters in cosmology. The collaboration has now released three years' worth of data, which covers an area of sky three times larger than their previous release [1]. The new analysis supports the standard cosmological model, with the measurements having unprecedented precision.

The main DES observations cover three things: galaxy clustering (the tendency of galaxies to appear close together in the sky), cosmic shear (the distortions in galaxy shape caused by gravitational lensing), and a combination of clustering and shear. Unlike other surveys, DES does all of its cosmic eyeballing from one telescope in Chile. This all-inclusive approach avoids potential pitfalls that can arise from combining measurements taken by different instruments or with different procedures.

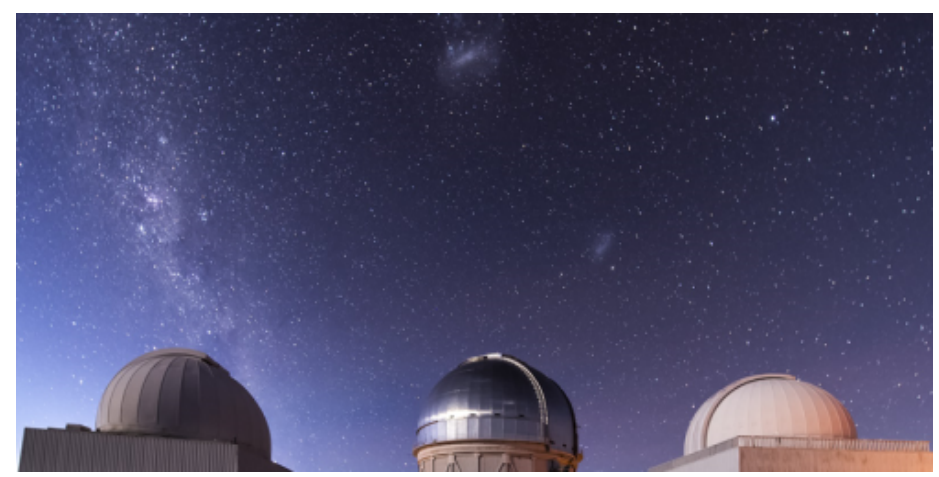

Credit: Reidar Hahn/Fermilab
In 2018, the DES Collaboration released its first-year observations, which covered $1514 \mathrm{deg}^{2}$ of sky (see Viewpoint: Weak Lensing Becomes a High-Precision Survey Science). The new three-year observations cover nearly $5000 \mathrm{deg}^{2}$, which is about one eighth of the whole sky. An updated data analysis resulted in a factor of 2 increase in the signal-to-noise ratio. The inferred values for cosmological parameters, such as the amplitude of matter clustering and the dark energy equation of state, agree with those predicted by the standard model of cosmology, in which dark energy is described by a cosmological constant. The uncertainty in the key parameters was less than $3 \%$, giving very little wiggle room to alternative theories.

Michael Schirber is a Corresponding Editor for Physics based in Lyon, France.

\section{REFERENCES}

1. T. M. C. Abbott et al. (DES Collaboration), "Dark Energy Survey Year 3 results: Cosmological constraints from galaxy clustering and weak lensing," Phys. Rev. D 105, 023520 (2022). 